\title{
Entwicklung eines taktilen Mikrotaster-Messystems für Hochgeschwindigkeitsmessung von Form, Rauheit und mechanischen Eigenschaften
}

\author{
Michael Fahrbach, Erwin Peiner \\ Institut für Halbleitertechnik (IHT) und Laboratory for Emerging Nanometrology (LENA), Technische \\ Universität Braunschweig, Braunschweig, Deutschland \\ m.fahrbach@tu-braunschweig.de, e.peiner@tu-braunschweig.de
}

\section{Zusammenfassung}

Im Rahmen eines europäischen EMPIR-Projekts soll das Messprinzip der Kontaktresonanzspektroskopie auf $5 \mathrm{~mm} \times 200 \mu \mathrm{m} \times 50 \mu \mathrm{m}(L \times B \times D)$ große, piezoresistive Tastsensoren angewandt werden. Dieses Messverfahren ist im Bereich der Rasterkraftmikroskopie bereits etabliert. Da die hier verwendeten Sensoren jedoch deutlich größer sind als im Bereich der Rasterkraftmikroskopie üblich, sind einige der dort geltenden Vereinfachungen nicht mehr anwendbar.

In dieser Arbeit werden Verfahren vorgestellt, die die Auswertung von Messwerten der verwendeten Sensoren verbessern, indem Schwingungsmodelle systematisch angepasst werden. Dazu gehören die Berücksichtigung der Luftdämpfung, von frequenzabhängigem Verhalten der Anregung sowie Nichtlinearitäten der Messbrücke.

Keywords: Mikrotaster, Cantilever-Sensor, Kontaktresonanzspektroskopie, Modellierung, Messdatenauswertung

\section{Einleitung}

Im Zuge der fortwährenden Digitalisierung der industriellen Fertigung werden neuartige Sensoren und Messverfahren benötigt, die Hochgeschwindigkeitsmessungen von Form, Rauheit und mechanischen Eigenschaften an Werkstücken und Werkzeugmaschinen während der Produktion ermöglichen [1]. Taktile Mikrotaster zeigen hier viel Potential, da sie genutzt werden können, um Oberflächen mit Geschwindigkeiten bis zu $15 \mathrm{~mm} / \mathrm{s}$ abzutasten [2]. Um solche Sensoren weiterzuentwickeln, wird ein europäisches EMPIR-Projekt finanziert [3]. Eines der Ziele dieses Projekts ist die Entwicklung eines Hochgeschwindigkeits-Messsystems zur Messung der oben genannten Größen.

Bei den hier verwendeten Sensoren handelt es sich um Silizium-Cantilever-Sensoren mit einer integrierten piezoresistiven Wheatstone'schen Messbrücke und einer Tastspitze nahe ihrem freien Ende der Firma CiS Forschungsinstitut für Mikrosensorik $\mathrm{GmbH}$, Erfurt vom Typ CAN50-2-5 [4]. Die geometrischen Abmessungen dieser Sensoren sind in Tabelle 1 aufgeführt.
Tab. 1: Nominelle Geometrie des Mikrotasters.

\begin{tabular}{|c|c|c|}
\hline Parameter & Symbol & $\begin{array}{c}\text { Nomineller } \\
\text { Wert }\end{array}$ \\
\hline Länge & $L$ & $5 \mathrm{~mm}$ \\
\hline Position der Spitze & $L_{1}$ & $4,9 \mathrm{~mm}$ \\
\hline Breite & $w$ & $200 \mu \mathrm{m}$ \\
\hline Dicke & $b$ & $50 \mu \mathrm{m}$ \\
\hline
\end{tabular}

\section{Messaufbau und Theorie}

Wie in Abbildung 1 dargestellt, werden die Sensoren auf sogenannten Mikrotasterträgern montiert.

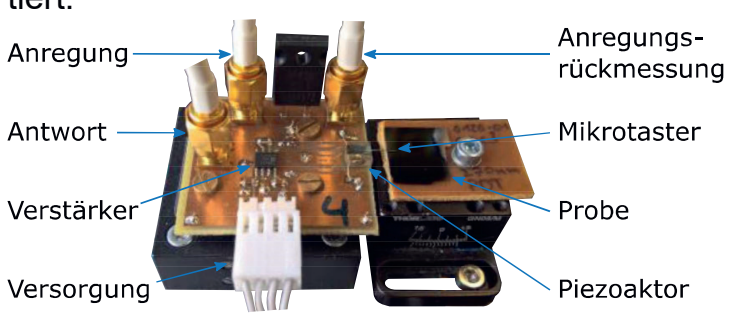

Abb. 1: Übersicht eines Mikrotasterträgers neben einer zu untersuchenden Probe.

Dabei handelt es sich um kleine Platinen, die einen Piezoaktor (Miniatur-Piezoaktor, 
$5 \times 5 \times 2 \mathrm{~mm}^{3}$, PL 055.30 PICMA®Chip Actuator, www.piceramic.de) zur dynamischen Anregung des Tasters, und einen Vorverstärker (Chip-Vorverstärker, AD8421, Analog Devices Inc.) zur Messung der Ausgangsspannung der Wheatstone'schen Messbrücke bereitstellen. Zur Anbindung an eine Messelektronik sind drei SMA-Buchsen vorgesehen, über die alle nötigen Signale abgegriffen werden können. Zusätzlich ist eine Pinleiste zur Spannungsversorgung der Bauteile verbaut.

Der Mikrotasterträger ist auf einem Positioniersystem, dargestellt in Abbildung 2, montiert. So kann er in der $x y$-Ebene über Positionierschrauben und in der $y z$-Ebene über Piezotische (PI P-518.ZCD und PI P-621.1CD, Physik Instrumente (PI) GmbH \& Co.KG, www.physikinstrumente.de) verfahren werden. Neben dem Mikrotasterträger angeordnet kann eine Probe auf einem $x y z$-Positionierer angebracht werden.

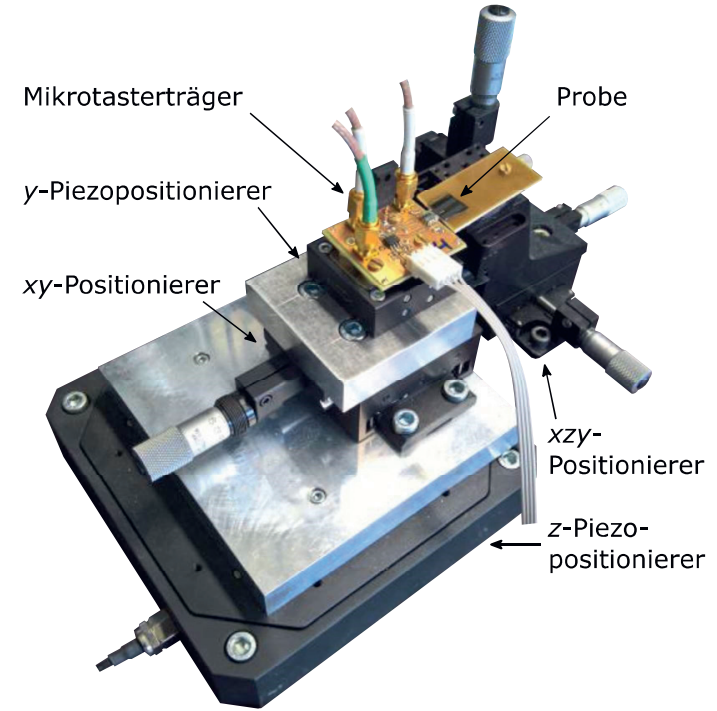

Abb. 2: Messtisch mit Mikrotasterträger und Probe. Die Spitze des Mikrotasters ist in Kontakt mit der Probe, die auf einem weiteren Positionierer befestigt ist.

Während einer Messung wird die Probe zunächst über die Positionierschrauben an den Taster herangeführt. Daraufhin wird der Taster mit dem z-Piezotisch an die Probe angenähert, bis Kontakt zwischen Tastspitze und Probenoberfläche hergestellt ist und der Cantilever beginnt sich zu biegen. Diese Bewegung wird so lange fortgeführt, bis die Kraft, die der Taster, entsprechend dem Produkt seiner Auslenkung und Steifigkeit auf die Probe ausübt, einen vorgegebenen Wert erreicht hat. Sobald dies geschehen ist, wird der Mikrotaster an seiner Basis durch den verbauten Piezoaktor in eine outof-plane-Biegeeigenschwingung angeregt.

Wie in Abbildung 3 dargestellt interagiert der Taster mit der Probenoberfläche, wodurch die
Resonanzfrequenzen und -amplituden, im Vergleich zum freischwingenden Sensor, zu höheren Werten verschoben bzw. verringert werden.

\section{Modellierung des gemessenen Verhaltens}

In [5, S. 55-58] stellt Rabe ein Modell auf, das das Verhalten von AFM-Sensoren in Kontakt mit Probenoberflächen unter Annahme verschiedener Randbedingungen und Vereinfachungen beschreibt. In ihm werden Länge, Breite, Dicke, Dichte und Elastizitätsmodul des Cantilevers, Position, Masse und Masseschwerpunkt der Tastspitze, Kontaktsteifigkeit und Dämpfung sowohl senkrecht als auch parallel zur Oberfläche sowie der Winkel zwischen Cantilever und Probenoberfläche berücksichtigt.

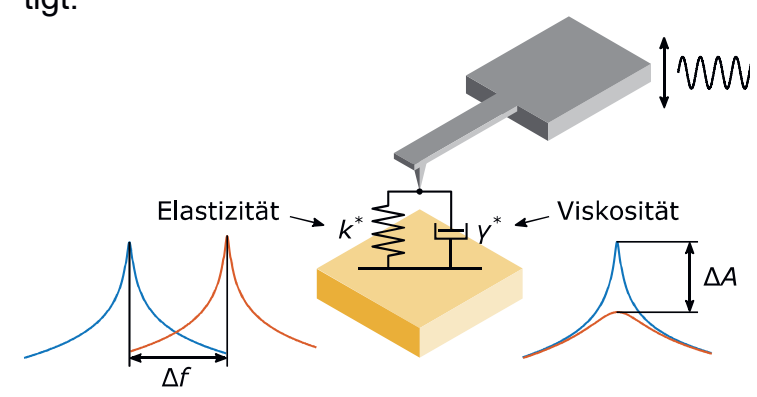

Abb. 3: Messprinzip der Kontaktresonanzspektroskopie. Der Sensor wechselwirkt mit der Probe, wodurch Resonanzfrequenz und -amplitude zu höheren Werten verschoben (links) bzw. verringert (rechts) werden (nach [6]).

Dieses Model wird nun an den Messaufbau und die Sensoren dieser Arbeit angepasst. So ist hier die Abweichung von einer senkrecht zur Probenoberfläche wirkenden Antastkraft, d. h. der Winkel zwischen Sensor- und Probenoberfläche mit ca. $1^{\circ}$ vernachlässigbar gering. In diesem Fall hat das Feder-Dämpfer-Glied parallel zur Oberfläche keine Auswirkung auf die Schwingung und kann somit vernachlässigt werden. Stattdessen muss aber die Luftdämpfung berücksichtigt werden, da sie sich drastisch auf die Resonanzgüte auswirkt [7] und somit zur Auswertung gemessener Amplituden unabdingbar ist. Des Weiteren wird entsprechend des Messaufbaus die Schwingungsanregung an der Basis des Sensors in das Modell aufgenommen.

Das Ergebnis ist ein Schwingungsmodell, das auf Grundlage physikalischer Zusammenhänge das Verhalten aller out-of-plane-Schwingungen des Mikrotasters beschreibt. Wird dieses Modell nun durch die Sensitivitäten des Sensors und des Piezoaktors aus den jeweiligen Datenblättern erweitert und mit Messungen verglichen, zeigen sich schnell einige Probleme: Einerseits 
kann das Modell die gemessenen Resonanzamplituden ohne Kenntnis der Luftdämpfung nicht nachbilden, andererseits können gemessene Resonanzfrequenzen, sowohl freischwingend als auch in Kontakt mit der Probe, durch das Modell nicht exakt reproduziert werden. Es ist somit nötig, so viele Parameter des Modells wie möglich zu überprüfen und anhand von Referenzmessungen so anzupassen, dass das Modell ein gemessenes Verhalten möglichst vollständig wiedergibt.

\section{Kalibrierung des Schwingungsmodells}

Die erste zu überprüfende Größe ist die Empfindlichkeit des Sensors. Diese wird gemessen, indem der Sensor quasistatisch gegen einen harten, nicht verformbaren Probenkörper, wie z. B. einen Siliziumchip, ausgelenkt wird, während gleichzeitig seine Position und Ausgangsspannung aufgezeichnet werden. Ein entsprechendes Messergebnis ist in Abbildung 4 dargestellt.

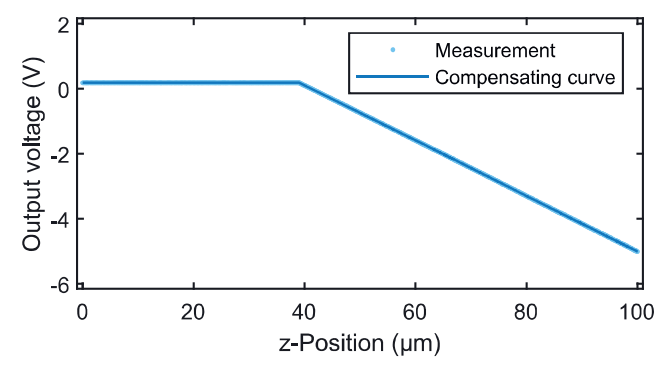

Abb. 4: Kennlinie der statischen MikrotasterAuslenkung. Gemessene Werte (Punkte) und Ausgleichskurve (Linie) sind nicht getrennt sichtbar. Eine konstante Ausgangsspannung liegt vor, bis der Sensor bei $z \approx 39 \mu \mathrm{m}$ Kontakt mit der Probe herstellt. Danach fällt die gemessene Spannung nahezu linear ab. Die Steigung hier entspricht der Empfindlichkeit.

Laut Datenblatt sollte die Ausgangsspannung des Mikrotasters in dieser Messung mit ca. $85 \mathrm{mV} / \mu \mathrm{m}$ abfallen, sobald Kontakt hergestellt ist. Der gemessene Verlauf der Empfindlichkeit in Abhängigkeit von der Cantileverauslenkung, dargestellt in Abbildung 5, weicht davon jedoch um bis zu 1,5 mV/ $\mu \mathrm{m}$ ab.

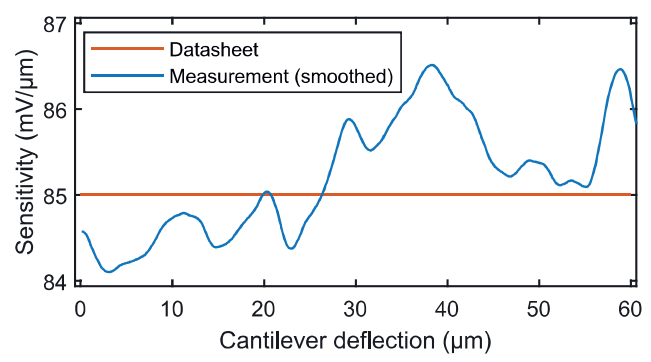

Abb. 5: Empfindlichkeit des Sensors in Abhängigkeit seiner Auslenkung. Wert laut Datenblatt (rot) und geglätteter gemessener Verlauf (blau).

Die mittlere gemessene Empfindlichkeit des Sensors liegt mit ca. 85,2 $\mathrm{mV} / \mu \mathrm{m}$ nahe an dem nominellen Wert, es zeigt sich jedoch auch ein Anstieg der Empfindlichkeit mit der Auslenkung. Die gemessene Auslenkungsabhängigkeit der Empfindlichkeit muss im Modell berücksichtigt werden.

Nachdem die Empfindlichkeit des Sensors bestimmt ist, werden Messungen der freischwingenden out-of-plane-Schwingungsmoden durchgeführt. Abbildung 6 zeigt beispielsweise die erste out-of-plane-Schwingungsmode. Hier können über die jeweilige Resonanzfrequenz, Resonanzgüte und Resonanzamplitude Informationen über Cantilever-Geometrie, Luftdämpfung und Empfindlichkeit der Anregung erlangt werden.

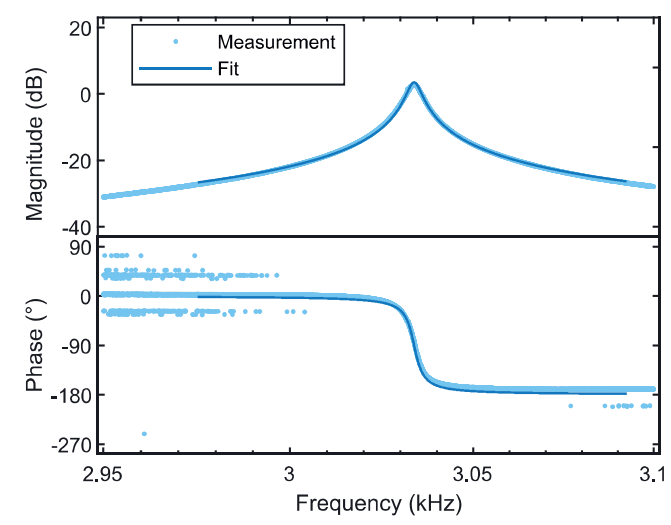

Abb. 6: Amplituden- (oben) und Phasengang (unten) des freischwingenden Sensors gemessen nahe der ersten outof-plane-Schwingungsmode: gemessener Verlauf (Punkte) und Fit des Schwingungsmodells an die Messwerte (Linie).

Zunächst werden die gemessenen Resonanzfrequenzen genutzt, um die nominelle Geometrie des Cantilevers zu überprüfen. Da Länge und Breite des Cantilevers in der Fertigung durch Lithographie sehr präzise eingestellt werden können, ist davon auszugehen, dass hier nur geringe Abweichungen zwischen Datenblatt und tatsächlichen Werten bestehen. Vielmehr sind die Abweichungen zwischen den Resonanzfrequenzen des Modells und den gemessenen Werten durch eine Abweichung der tatsächlichen Dicke des Cantilevers vom Nominalwert zu erklären.

Wie Abbildung 7 zeigt, liegen die Resonanzfrequenzen des Modells unter Annahme der nominellen Geometrie stets unter den gemessenen 
Werten. Durch Erhöhung der Dicke des Cantilevers im Modell auf ca. 55,3 $\mu \mathrm{m}$ nehmen die Abweichungen zwischen Modell und Messungen auf weniger als 3\% ab. Diese verbleibenden Abweichungen können durch Idealisierungen des Modells erklärt werden, die der Realität nur näherungsweise entsprechen. Um dies zu berücksichtigen wird eine Ausgleichskurve durch die verbleibenden Abweichungen gelegt und zur Umskalierung der Frequenzachse verwendet. So bildet das Modell alle gemessenen Resonanzfrequenzen nach. Dies ist für die weitere Auswertung wichtig, da es andernfalls nicht möglich wäre das Modell an gemessene freischwingende Resonanzmoden zu fitten.

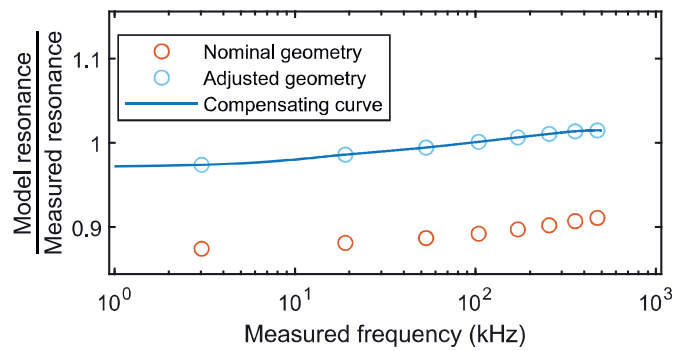

Abb. 7: Modell-Resonanzfrequenz bezogen auf gemessene Resonanzfrequenz für die ersten acht out-of-planeSchwingungsmoden eines Cantilevers unter Annahme der nominellen Sensorgeometrie laut Datenblatt ([4], rote Kreise) und nach Anpassung der Cantileverdicke (blaue Kreise) mit einer Ausgleichskurve (blaue Linie), die in die weitere Modellrechnung eingeht.

Nachdem sichergestellt ist, dass die gemessenen Resonanzfrequenzen mit denen des Modells übereinstimmen, kann das Modell, wie in Abbildung 6 zu sehen ist, an die Messungen gefittet werden. Für jede Resonanzmode wird so jeweils ein Wert der Luftdämpfung und einer der Empfindlichkeit der Anregung bestimmt.

Die resultierenden Werte der Luftdämpfung sind in Abbildung 8 aufgetragen. Es zeigt sich ein monotoner Verlauf, in dem die Luftdämpfung mit steigender Frequenz steigt. Da Werte der Luftdämpfung a priori nicht bekannt sind [5], können hieraus jedoch keine weiteren Schlüsse gezogen werden.

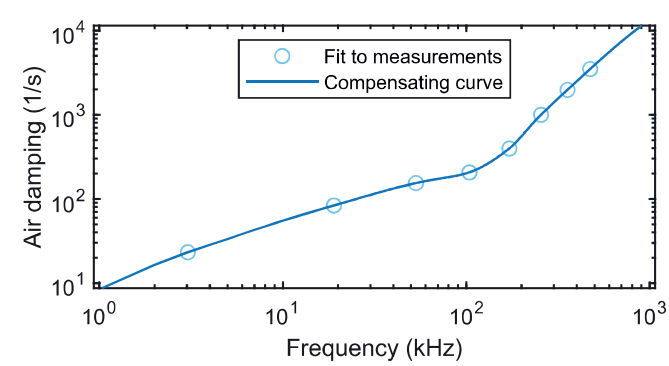

Abb. 8: Frequenzverlauf der Luftdämpfung: Bestimmt durch Anpassungen des Schwingungsmodells an Messungen des freischwingenden Sensors in den Frequenzbereichen um die ersten acht out-of-plane-Schwingungsmoden herum.

Im nächsten Schritt werden Resonanzmoden des Sensors in Kontakt mit einer Probenoberfläche aufgenommen. Ein Beispiel für eine solche Messung ist in Abbildung 9 dargestellt.

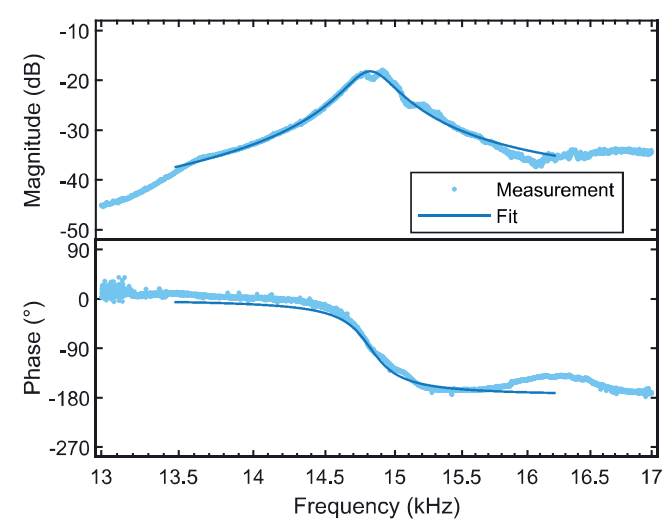

Abb. 9: Amplituden- (oben) und Phasen-Frequenzgang (unten) bei Kontakt des Sensors mit einer Probe in der Umgebung der ersten out-of-plane-Schwingungsmode: gemessener Verlauf (Punkte) und Fit des Schwingungsmodells an die Messwerte (Linien).

Aus diesen Kurven werden durch Anpassung anschließend jeweils ein Wert für die Kontaktsteifigkeit, die Dämpfungskonstante und die Empfindlichkeit der Anregung bestimmt. Werden mehrere Moden in Kontakt unter gleichen Bedingungen untersucht, so sollte die bestimmte Kontaktsteifigkeit für alle Moden identisch sein [8]. Ist dies nicht der Fall, so muss die Position der Tastspitze $L_{1}$ im Modell angepasst werden.

Aus den Messungen der freischwingenden Resonanzmoden und der Kontaktresonanzmoden kann nun der Frequenzverlauf der Empfindlichkeit des Piezoaktors bestimmt werden. Wie in Abbildung 10 dargestellt, liegen die bestimmten Werte sämtlich unter dem nominellen Wert aus 
dem Datenblatt. Dies ist möglicherweise durch Schwingungen der Mikrotasterträger-Platine zu erklären. Es ist jedoch unklar, ob dieser Effekt allein die Abweichungen von bis zu zwei Größenordnungen erklärt. Trotz verbleibender Unklarheiten führt die Berücksichtigung der aus den Anpassungen ermittelten Korrekturwerte dazu, dass das Modell die Messkurven besser nachbildet, weshalb darauf nicht verzichtet wird.

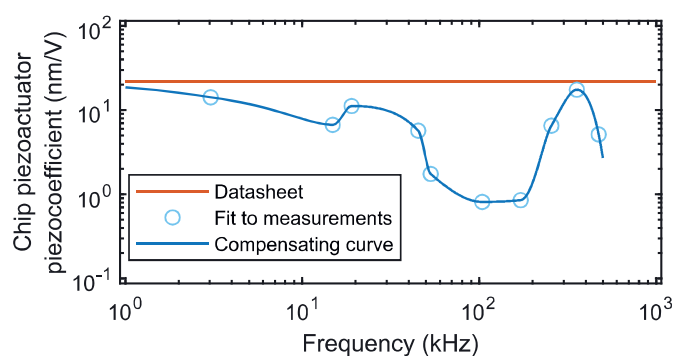

Abb. 10: Frequenzverlauf des Piezokoeffizienten des verwendeten Piezoaktors: Wert aus Datenblatt ((Miniatur-Piezoaktor, $\quad 5 \times 5 \times 2 \mathrm{~mm}^{3}, \quad$ PL 055.30 PICMA®Chip Actuator, www.piceramic.de), rot), Verlauf bestimmt durch Anpassungen des Schwingungsmodells an Messungen nahe den ersten acht out-of-planeSchwingungsmoden bei freischwingendem Sensor und der ersten beiden out-of-plane-Schwingungsmoden bei Kontakt mit einer Probe (blaue Kreise) mit Ausgleichskurve (blaue Linie).

Der letzte Schritt der Kalibrierung ist die Bestimmung der Position der Tastspitze $L_{1}$ entlang der Cantileverachse. Die Notwendigkeit dieses Schrittes ist nicht durch Abweichungen zwischen nominellen Werten und realem Sensor, sondern durch Idealisierungen im Modell zu erklären, die der Realität nur näherungsweise entsprechen [8]. Um dies zu berücksichtigen, werden mindestens zwei Kontaktresonanzmoden des Tasters in Kontakt mit der gleichen Probe unter gleichen Bedingungen aufgenommen. Anschließend werden verschiedene Werte für $L_{1}$ angesetzt, das Modell wird mit allen hier beschriebenen Methoden auf diese Spitzenposition kalibriert und die durch Anpassung bestimmten Kontaktsteifigkeiten der gemessenen Resonanzmoden werden verglichen. Wird dies für eine ausreichend hohe Anzahl an Spitzenpositionen durchgeführt, ergibt sich ein Verlauf entsprechend Abbildung 11.

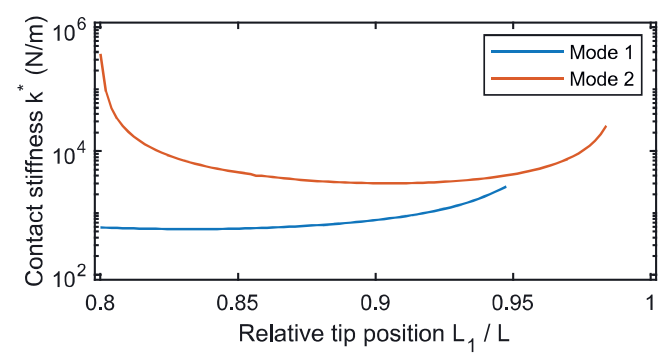

Abb. 11: Kontaktsteifigkeit in Abhängigkeit von der relativen Position der Spitze bezogen auf die Länge des Cantilevers, berechnet aus den gemessenen Resonanzfrequenzen in der ersten (blau) und zweiten (rot) out-of-planeSchwingungsmode. Sobald $L_{1} / L$ den Wert 0,95 im Fall der ersten bzw. 0,98 im Fall der zweiten Mode überschreitet, kann die gemessene Resonanzfrequenz nicht mehr nachgebildet werden.

Im Fall der ersten Kontaktresonanzmode führt die Verschiebung der Tastspitze in Richtung des freien Endes zu steigenden Kontaktsteifigkeiten. Überschreitet $L_{1} / L$ den Wert 0,98 , so kann das Modell die gemessene Resonanzfrequenz, wie in Abbildung 9 dargestellt, nicht mehr nachbilden. Bei Betrachtung der zweiten Kontaktresonanzmode fällt die Kontaktsteifigkeit zu Beginn und steigt später wieder an. Hier kann die gemessene Resonanzfrequenz ab einer relativen Spitzenposition von 0,98 nicht nachgebildet werden. Mit der nominellen Spitzenposition $L_{1}=4,9 \mathrm{~mm} \quad\left(L_{1} / L=0,98\right) \quad$ kann folglich nur die zweite gemessene Resonanzfrequenz reproduziert werden.

Entgegen der Vermutung gemäß [8] schneiden sich beide Kurven jedoch nicht. Deshalb wird nicht der Schnittpunkt sondern der Punkt der geringsten Abweichung zwischen beiden Kurven für die Bestimmung der Spitzenposition verwendet. Dieser liegt bei $L_{1}=4,74 \mathrm{~mm}\left(L_{1} / L=\right.$ 0,948 ).

Das kalibrierte Modell kann nun genutzt werden um Messungen auszuwerten. In Abbildung 12 ist beispielsweise das Ergebnis von Messungen an einer dünnen Polymerschicht auf einem Siliziumsubstrat dargestellt. In diesen Messungen wurde die Resonanzfrequenz der ersten out-ofplane-Schwingungsmode bei verschiedenen Antastkräften untersucht. Die Antastkraft wurde anschließend über die in Abbildung 4 dargestellte Kurve in die Deformation der Probe umgerechnet. Mit steigender Deformation wird zunehmend der Einfluss des Siliziumsubstrats unter der Polymerschicht gemessen, wodurch die Kontaktsteifigkeit steigt. 


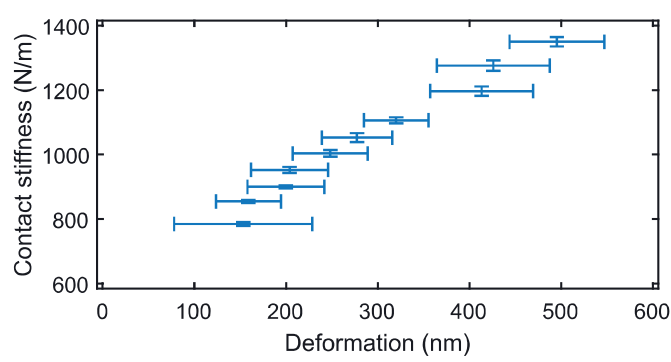

Abb. 12: Kontaktsteifigkeit in Abhängigkeit von der Deformation der Probe, gemessen in der ersten out-of-planeSchwingungsmode an einer $1400 \mathrm{~nm}$ dünnen Schicht von mr-NIL210 (micro resist technology $\mathrm{GmbH}$ ) Nanoimprintlack auf einem Siliziumsubstrat.

\section{Fazit und Ausblick}

In dieser Arbeit wurde ein KontaktresonanzSchwingungsmodell aus der Rasterkraftmikroskopie an einen deutlich größeren Cantileversensor angepasst. In diesem Zuge wurde eine Methode vorgestellt, das Modell auf Basis von Referenzmessungen systematisch zu kalibrieren, sodass Amplituden-/Phasenfrequenzgänge im Bereich der resonanten out-of-planeSchwingungsmoden möglichst genau nachgebildet werden. Dafür wurden Nichtlinearitäten des Sensors, Abweichungen der Sensorgeometrie vom Datenblatt, Auswirkungen der Luftdämpfung sowie Frequenzabhängigkeiten der Anregung berücksichtigt. Die vorgestellte Prozedur bildet die Grundlage dafür, Kontaktresonanz-Messungen an dünnen Schichten auf Werkstücken präzise und reproduzierbar auswerten zu können. Als Anwendungsbeispiel wurde eine ausgewertete Messung an einer dünnen Polymerschicht aufgeführt.

In weiteren Analysen müssen allerdings noch verbleibende Unschlüssigkeiten geklärt werden: Einerseits muss das Ausmaß der Frequenzabhängigkeit des Piezoaktors untersucht werden, um zu überprüfen, ob die hier bestimmten Werte der Realität entsprechen. Andererseits müssen weitere Kontaktresonanzmessungen an Referenzproben durchgeführt werden, um die durch die Position der Tastspitze gegebene effektive Cantileverlänge für die relevanten Schwingungsmoden konsistent zu bestimmen.

Daraufhin müssen die Kontaktsteifigkeiten und Dämpfungskonstanten, die mit dem in dieser Arbeit beschriebenen Modell an Dünnschichtproben bestimmt werden, mit Hilfe von Referenz-Messverfahren auf physikalische Größen wie das (komplexe) Elastizitätsmodul zurückgeführt werden.

\section{Finanzierung}

This project has received funding from the EMPIR programme co-financed by the Participating States and from the European Union's Horizon 2020 research and innovation programme under no. 17IND05 MicroProbes.

\section{Literaturnachweis}

[1] Hofmann, E., Rüsch, M. Industry 4.0 and the current status as well as future prospects on logistics. Computers in Industry 89, 23-34 (2017). https://doi.org/10.1016/j.compind.2017.04.002

[2] Wasisto, H. S.; Doering, L.; Brand, U.; Peiner, E. Ultra-high-speed cantilever tactile probe for high-aspect-ratio micro metrology. In 2015 Transducers - 2015 18th International Conference on Solid-State Sensors, Actuators and Microsystems (TRANSDUCERS), Anchorage, AK, USA, 21-25 June 2015, pp. 1061-1064. IEEE. https://doi.org/10.1109/transducers.2015.71811 09

[3] EMPIR Project "17IND05 MicroProbes Multifunctional ultrafast microprobes for on-themachine measurements", https://www.ptb.de/empir2018/microprobes/

[4] https://www.cismst.org/loesungen/mikrotastspitzen/. [Zugriff: 29. 04. 2019]

[5] Rabe, U. Atomic force acoustic microscopy. Applied Scanning Probe Methods II 37-90 (2006). Springer Berlin Heidelberg

[6] Kocun, M., Ohler, B. Exploring Nanoscale Viscoelastic Properties. Imaging \& Microscopy 15 No. 4 22-24 (2013)

[7] Lübbe. J., Schnieder, H., Reichling, M. Pressure Dependence of the Q-factor of Cantilevers Used for NC-AFM. E-Journal of Surface Science and Nanotechnology 9 30-33 (2011)

[8] Hurley, D. C. Contact resonance force microscopy techniques for nanomechanical measurements. Applied Scanning Probe Methods XI 97-138 (2009). Springer Berlin Heidelberg 